\title{
INITIAL PROVISIONING AND SPARE PARTS INVENTORY NETWORK OPTIMISATION IN A MULTI MAINTENANCE BASE ENVIRONMENT
}

\author{
Peter Lendermann \\ Annamalai Thirunavukkarasu \\ Malcolm Yoke Hean Low \\ D-SIMLAB Technologies Pte Ltd \\ 8 Jurong Town Hall Road \\ \#30-04 JTC Summit \\ SINGAPORE 609434
}

\author{
Leon F. McGinnis \\ School of Industrial and Systems Engineering \\ Georgia Institute of Technology \\ Atlanta, GA 30332-0205 \\ U.S.A.
}

\begin{abstract}
Aviation spare parts provisioning is a highly complex problem. Traditionally, provisioning has been carried out using a conventional Poisson-based approach where inventory quantities are calculated separately for each part number and demands from different operations bases are consolidated into one single location. In an environment with multiple operations bases, however, such simplifications can lead to situations in which spares - although available at another airport - first have to be shipped to the location where the demand actually arose, leading to flight delays and cancellations. In this paper we demonstrate how simulation-based optimisation can help with the multi-location inventory problem by quantifying synergy potential between locations and how total service lifecycle cost can be further reduced without increasing risk right away from the Initial Provisioning (IP) stage onwards by taking into account advanced logistics policies such as pro-active re-balancing of spares between stocking locations.
\end{abstract}

\section{INTRODUCTION}

The problem of determining optimal quantities in an aviation spare parts inventory network is highly complex. Many larger airlines operate several hubs, especially for their short-haul fleets, and therefore have maintenance bases at multiple locations. In this setting, sophisticated decision support tools are required to determine optimal stocking quantities at all these maintenance bases.

Contemporary Initial Provisioning (IP) approaches assume that needs for spare parts are to be fulfilled at one single maintenance base. In a multi-location environment however, especially for critical-todispatch components (NO-GO items), this does not work because in case of a removal a replacement unit - although on stock at a different location - cannot necessarily be made available in time to avoid a technical delay or a flight cancellation at the location where the demand arose. In this kind of environment each aircraft of the fleet to be supported would have to be allocated to one of the maintenance bases, and then (at least for critical-to-dispatch components) IP exercises would have to be carried out separately for each maintenance base. This would imply that no synergy potential can be exploited between the individual maintenance bases, and hence IP quantities would go beyond what is considered economically viable.

In reality, however, synergy potentials do exist because often it takes a considerable amount of time to first remove an unserviceable unit from an aircraft before a replacement can actually be installed. Moreover, in many cases there is still time available until the affected aircraft has to be ready for dispatch. 


\section{Lendermann, Thirunavukkarasu, Low, and McGinnis}

This paper demonstrates through a realistic case study how simulation-based optimisation techniques can help in tackling the multi-location inventory problem. In particular, we show how:

- Spares requirements for a multi-location network are higher compared to a scenario where the entire demand is to be fulfilled at one single location;

- Synergy potentials between inventory locations, depending on delivery time requirements, can be quantified;

- Removal of planning constraints associated with risk mitigation policies can also help in keeping investments low;

- Re-balancing of inventory between stockholding nodes to reflect systemwide availability can help reduce total service lifecycle cost without increasing risk right away from the IP stage onwards.

The remainder of this paper is organised as follows: Section 2 is dedicated to a review of related literature. Following an introduction into of D-SIMSPAIR, the software package used for the analysis described in this paper in Section 3, and an overview of the associated case study in Section 4, the analysis of the above-mentioned aspects of spare parts inventory network optimisation in a multi maintenance base environment is described in Section5. Finally, the main conclusion of this paper is stated in Section 6.

\section{RELATED WORK}

Simulation is a powerful tool for evaluating different operation strategies in complex real world problems such as the provisioning of inventory for airlines. The use of simulation to evaluate and optimise airline operations was first proposed by Gunn (1964), where the objectives of the study were on optimising airline route and passenger capacity. Recent work by Lee et al. (2003) developed a discrete event simulation research tool, SimAir, to study the robustness of airline schedules and to obtain a feasible schedule in the event of a disruption. Neither of these works considered the problem of inventory provisioning of spares for ensuring that airlines maintain a high service level for their aircraft.

One specific branch of studies that looks at the aerospace inventory provisioning problem adopts the Virtual Warehouse (VW) concept. Among the first to describe and point out the relevance of this concept for a business model that exploits the use of real-time global visibility of geographically disperse logistics assets such as inventory and vehicles and dynamically moves these assets between locations to achieve operation efficiency, cost reduction, and high service level comparable to that achieved in a business model that uses only inventory from local warehouse facilities were Landers et al. (2000).

Fung et al. (2005) further extended the VW concept to production logistics where a simulation model for inventory planning, a production optimisation tool and a knowledge base were integrated into a decision support tool for refining inventory planning so as to streamline production planning and control to anticipate any abrupt change in the product demand. In the work by Lye and Chan (2007), the concept of virtual warehouse was extended to study aerospace inventory provisioning strategies through a simulation model that involved storing spares inventory at different airports and shipping them as needed to other airports when needs arose based on real-time information. Lee et al. (2007) described the integration of a multi-objective evolutionary algorithm (MOEA) with multi-objective computing budget allocation (MOCBA) method for the multi-objective simulation optimisation problem on aircraft spare parts allocation.

In the conventional Poisson-based inventory provisioning approach, the inventory quantities for each parts are determined separately (without reference to the cost of the parts) to meet the target service level (Rutledge 1997). The target service level for each part (and also the overall system service level) is often exceeded due to the rounding of the inventory quantities. Airlines and MRO providers typically commit to a minimum overall service level for a group of parts instead of individual service level for each part. Thus, system level optimisation can be carried out to meet the required target service level by stocking more of the inexpensive parts and fewer of the more expensive parts. Although the achieved service levels for the individual inventory items will deviate from the target service level, the overall target service 


\section{Lendermann, Thirunavukkarasu, Low, and McGinnis}

level is still met. Both Lye and Chan (2007) and Lee et al. (2007) did not consider the interdependencies among different groups of parts of the same level of criticality in their work.

In the work by MacDonnell and Clegg (2011, 2007), linear programming models are used to determine an optimum set of inventory levels for a set of parts that meets the overall target service level at the minimum cost. Note that in their study, only a single mainbase is considered and real-world requirements such as pooling of inventory at different airports by MRO provider serving different operators with different delivery time commitments for different groups of components, and also different logistics connectivity between airports are not considered.

The concept of pooling of spares between airlines was proposed by Kilpi and Vepsalainen (2004) to exploit the economies of scale and the savings potential of balanced inventory pooling arrangements among various airlines with geographically disperse maintenance bases. Lendermann et al. (2010) described the use of a simulation-based rotables inventory optimisation tool D-SIMSPAIR that is able to consider the underlying complexity of the flight network and the pooling potential of inventory in different stockholding locations to carry out optimisation across a group of several part numbers to determine optimised inventory levels with the minimum cost that satisfies a target service level. This paper extends the work described in Lendermann et al. (2010) by carrying out an in-depth analysis of an inventory optimisation for an airline with multiple maintenance bases where logistics flights based on actual realworld flight schedules are used to deliver spares to fulfill demands across the network of destination airports.

\section{SIMULATION TOOL USED FOR THIS STUDY}

The software package used for the analysis described in this paper is D-SIMSPAIR, an advanced domainspecific decision support tool for rotables and expendables inventory planning and optimisation in the aviation industry. It adopts a simulation-based optimisation approach. It has been designed for use either stand-alone or on top of an Enterprise Resource Planning system and has the capability to portray the dynamic attributes of a spare parts logistics network with high fidelity in a Discrete Event Simulation model. This is particularly important when it comes to making quantitative commitments, especially with regard to delivery time and schedule-dependent dispatch reliability in networks with multiple maintenance and inventory storage locations. Furthermore, it satisfies the need for integration between the underlying simulation model and a sophisticated domain-specific optimisation procedure to enable complex decisionsupport tasks and can thus unlock considerable value from component support contracts between an MRO service provider and airline customers, even for single stockholding node scenarios as illustrated in this paper.

The D-SIMSPAIR approach recognises that - regardless of individual part numbers $(\mathrm{P} / \mathrm{Ns})$ - the real business challenge is either to attain a required service levelat minimum cost or to maximise the service level for a given budget in the context of an entire component support contract, i.e., the combined set of $\mathrm{P} / \mathrm{Ns}$ on an aggregate level rather than for the individual $\mathrm{P} / \mathrm{Ns}$ one by one.

\section{CASE STUDY}

The case study portrays an (imaginary) airline with a principal maintenance base in Los Angeles (LAX) and two more maintenance bases in Dallas (DFW) and Washington-Dulles (IAD). The fleet comprises of 20 long-haul aircraft accumulating 4,800 flight hours per year, flying to eight international destinations, with ten aircraft operated from LAX and five each from DFW and IAD.

Table 1 lists a representative set of 10 NO-GO items used for the purpose of this analysis. The reliability of components is characterised by their MTBUR value (Mean Time Between Unscheduled Removals). The table also lists their unit price and the quantity per aircraft (QPA). The repair turnaround time (repair TAT) is assumed to be 30 days for all $\mathrm{P} / \mathrm{Ns}$ and also includes logistics transfer time to and from the repair station. 


\section{Lendermann, Thirunavukkarasu, Low, and McGinnis}

Table 1: Details of the components used for this analysis

\begin{tabular}{|l|rrrrrrrrrr|}
\hline Part Number & Part-01 & Part-02 & Part-03 & Part-04 & Part-05 & Part-06 & Part-07 & Part-08 & Part-09 & Part-10 \\
\hline $\begin{array}{l}\text { Unit Price(USD) } \\
\text { MTBUR }\end{array}$ & 45,000 & 50,000 & 60,000 & 150,000 & 8,000 & 200,000 & 45,000 & 5,000 & 150,000 & 15,000 \\
$\begin{array}{l}\text { flight hours) } \\
\text { QPA }\end{array}$ & 300,000 & 100,000 & 50,000 & 37,500 & 27,500 & 25,000 & 100,000 & 5,000 & 3,200 & 2,500 \\
& 1 & 1 & 1 & 1 & 1 & 1 & 10 & 1 & 1 & 1 \\
\hline
\end{tabular}

In case a spare unit has to be brought in from another maintenance base, it is assumed that logistics flights are available according to a realistic flight schedule between the respective airports with minimum flight duration as displayed in Table 2, and waiting times will be incurred as illustrated in Figure $\mathbf{1 .}$

Table 2: Average flight time between airports

\begin{tabular}{|c|l|ccc|}
\hline \multicolumn{2}{|c|}{$\begin{array}{c}\text { Average Flight } \\
\text { Time between } \\
\text { Airports }\end{array}$} & \multicolumn{3}{|c|}{ Destination } \\
\cline { 2 - 5 } Origin & LAX & - & $2 \mathrm{hrs} 45 \mathrm{mins}$ & $4 \mathrm{hrs} 35 \mathrm{mins}$ \\
\hline & DFW & $3 \mathrm{hrs}$ & - & $2 \mathrm{hrs} 35 \mathrm{mins}$ \\
& IAD & $5 \mathrm{hrs} 15 \mathrm{mins}$ & $3 \mathrm{hrs}$ & - \\
\hline
\end{tabular}

The cost incurred when carrying out such a shipment is assumed to be USD 1,000 per movement, and the annual inventory holding costs for a spare part is taken as $20 \%$ of the component unit price. The aggregate service level to be delivered to the airline is $95 \%$.

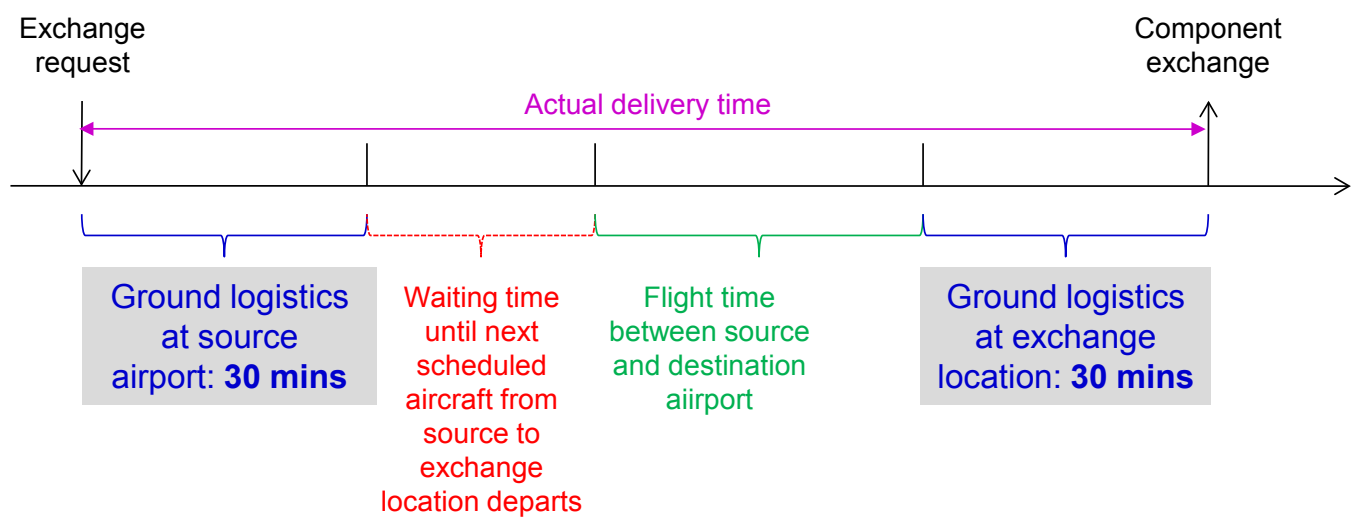

Figure 1: Timing for logistics flights

\section{ANALYSIS}

\subsection{IP at Multiple Maintenance Bases versus IP at Single Maintenance Base}

The analysis was carried out using D-SIMSPAIR. As shown in Figure 2 the total inventory required for a scenario with three independently operated maintenance bases LAX, DFW and IAD is considerably higher compared to the case where the entire spares demand for all 20 aircraft would have to be fulfilled at the principal maintenance base LAX. 


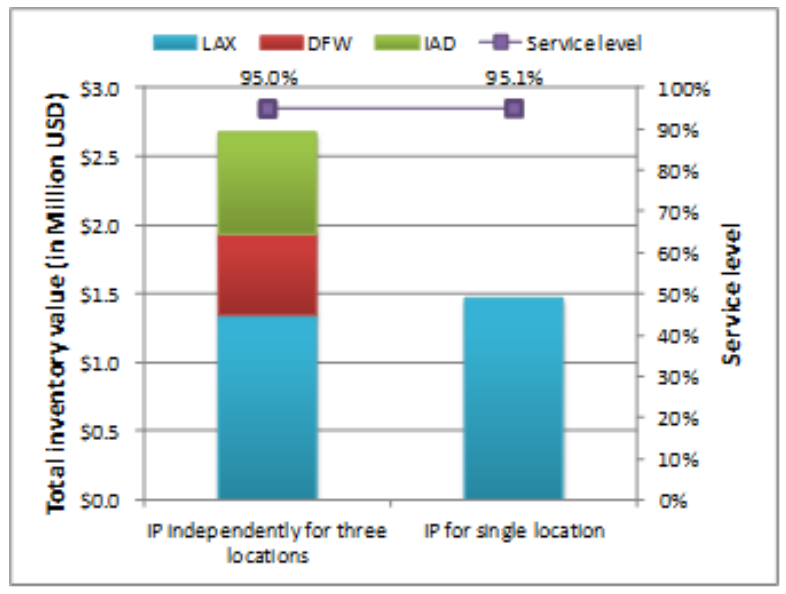

Figure 2: Comparison of IP requirements for single and multiple locations

\subsection{Synergy Potential between Maintenance Bases}

An important parameter for component support services is the delivery time commitment, i.e. the maximum time allowed between a component exchange request and its fulfillment (beyond which a demand would be considered "unfilled"). Since in reality the required delivery time often would not be immediate, it is to some extent possible to pool spares across several maintenance bases.

\subsubsection{Effect of Delivery Time Commitment on IP Requirements}

Figure 3 displays the value of total inventory required to achieve $95 \%$ service level for different delivery time commitments.

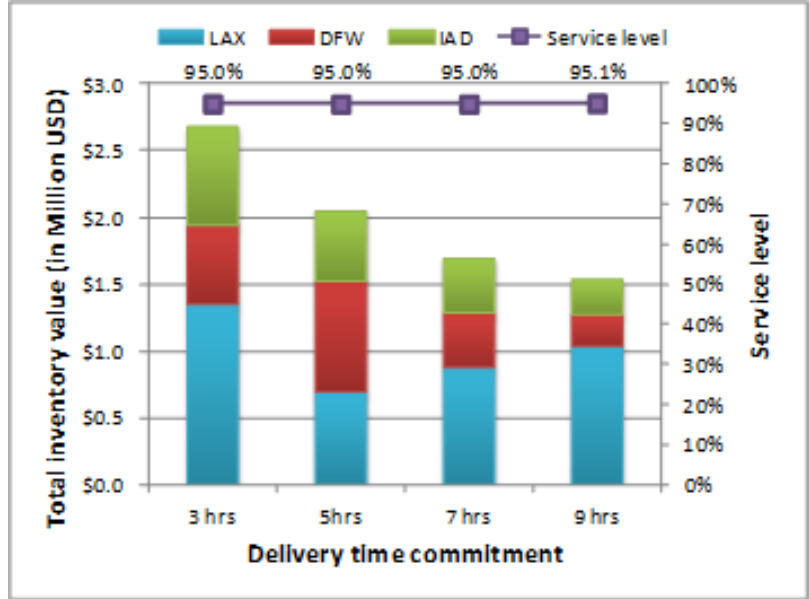

Figure 3: Effect of increasing delivery time commitment at maintenance bases on total inventory requirements

With a delivery time commitment of 3 hours pooling of spares between maintenance bases will basically not be possible under any circumstance as the minimum time required to move a spare unit (on a logistics flight) from one maintenance base and make it available at another is greater than the delivery time commitment. In this case the inventory distribution is equivalent to the case where IP is carried out independently for each maintenance base (see left column in Figure 2). 
When the delivery time commitment is increased to 5 hours pooling between DFW and the other two maintenance bases (IAD and LAX) will become possible in situations where a logistics flight is just ready to depart once a spare has been made available at DFW to fulfil a demand at LAX or IAD, resulting in a reduction of inventory requirements. In fact some of the LAX inventory is to be relocated to DFW and the value of inventory at DFW is even higher compared to LAX (despite more flight hours to be protected at LAX) as DFW now becomes the strategic location to serve all three maintenance bases.

With further increase of delivery time commitment to 7 hours more shipments between DFW and LAX or IAD will be feasible in both directions. On top of that, even shipments between LAX and IAD (and vice versa) will sometimes be possible now. As a result, 95\% service level can now be achieved with even less inventory. In fact, since sharing of inventory is now possible between all the maintenance bases, to reduce logistics cost some of the stock at DFW will be repositioned back to LAX where most flight hours are to be protected.

When the delivery time commitment is further increased to 9 hours, the total inventory value will decline even further. This reduction can be attributed to the increased chance of having a logistics flight available for an in-time delivery.

\subsubsection{Effect of Delivery Time Commitment on Service Level}

The synergy potential between the maintenance bases can also be tuned by adjusting service levels that can be guaranteed for existing inventory quantities across the maintenance bases according to different delivery time commitment values.

Figure 4 shows how with an increase in delivery time commitment the service level that can be promised increases for the same inventory configuration.

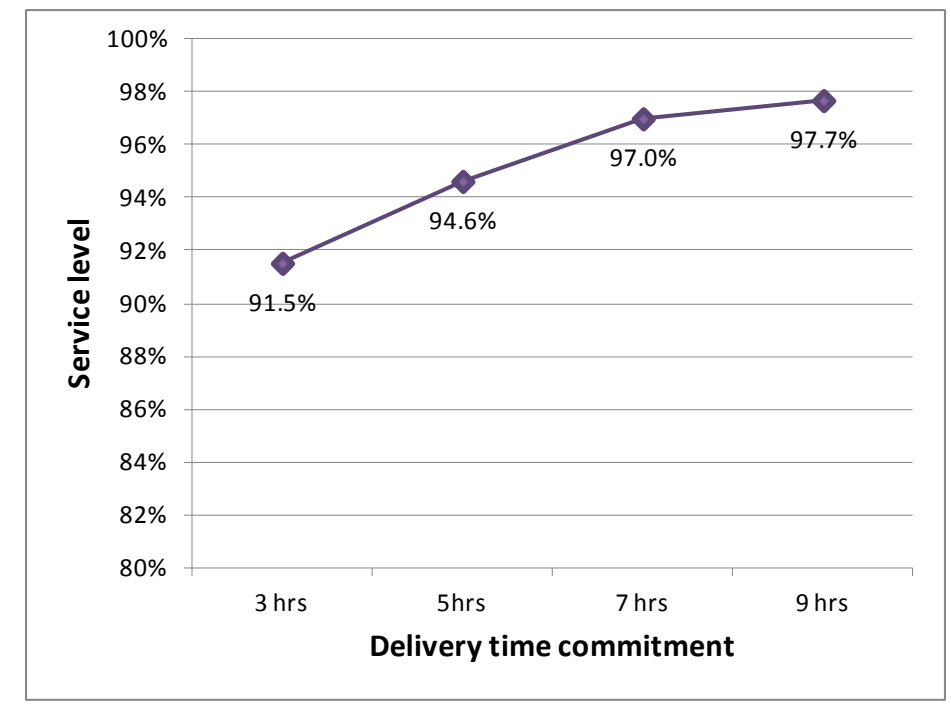

Figure 4: Effect of increasing delivery time commitment at maintenance bases on service level

Through the ability to simulate spare parts delivery cycle in a very realistic manner (to the extent that even the effect of the 'time of the day' can be taken into account), delivery time commitments can be fine-tuned part number by part number, depending on constraints imposed by the time to remove an unserviceable unit from the aircraft and to install a replacement as well as the effect on dispatch reliability. In this way synergy exploitation can be further enhanced to ensure that IP budgets are kept as low as possible without compromising service level. 


\subsection{Removal of Planning Constraints}

MRO supply chains are driven by unscheduled removals, i.e. the demand for spares is highly probabilistic in nature. It is therefore not uncommon to plan at least for one spare unit for all critical-to-dispatch components during an IP exercise (Minimum-1 constraint). At the same time, service levels are guaranteed not for each part number individually but on an aggregate level for groups of part numbers.

In this setting, as long as the aggregated risk can be managed appropriately it does not matter whether an 'Aircraft-On-Ground' (AOG) situation is caused by not being able to provide an expensive control unit or a cheap lighting component. With the usage of a tool incorporating a high-fidelity simulation approach, demands for components can be gauged much more accurately thereby avoiding the necessity to impose additional planning constraints.

In this setting, the removal of the Minimum-1 constraint can help reduce cost considerably without increasing risk as the number of expensive units would be reduced to zero and offset by having more of the cheaper components available. As shown in Figure 5, without the Minimum-1 constraint the total investment required for a scenario with $90 \%$ target service level would be reduced by about $10 \%$. For those part numbers where the recommended IP quantity is zero the provisioning lead time does not have a direct effect any more since all demands for those part numbers will result in no-fill events.

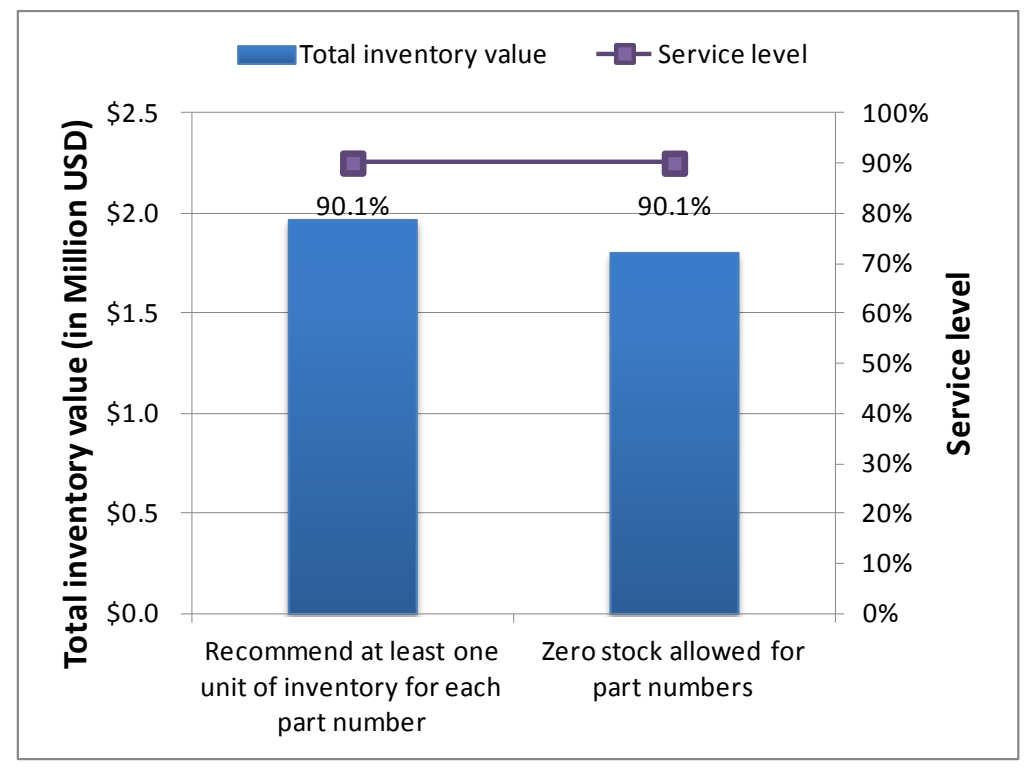

Figure 5: Effect of removing planning constraints on total inventory value

\subsection{Inventory Re-Balancing}

In a multi-location environment, in a situation where the number of spare units at a particular location has been depleted to zero, a pro-active transhipment of a spare unit from another location that still has one or more units available might be considered to minimise the risk of not being able to service subsequent demands at the same location until a serviceable unit returns from repair.

Obviously, such a re-balancing policy is not advisable for all part numbers since transhipments associated with re-balancing movements incur cost. Several factors determine whether for a particular part number re-balancing would actually be economically viable:

(1) Unit price: Re-balancing would not be a worthwhile policy for very cheap items as it would be better to stock more units right away. 
(2) Demand profile: Re-balancing to a particular location would not be carried out if the number of flights to be protected at that location is low.

(3) MTBUR: Re-balancing would also not be advisable for high reliability parts as the risk associated with not doing anything is very low.

(4) Repair turnaround time (TAT): Re-balancing would also not be worthwhile if the repair turnaround time is short, i.e. a few days only.

A simulation-based optimisation approach can portray the complex interdependencies between these effects and determine the economic viability of re-balancing for each part number, taking into account the above-listed factors. Table 3 shows the detailed cost profile for the case where re-balancing is considered as a decision variable and indicates for which part number it is an economically viable policy.

As shown in Figure 6, re-balancing decreases significantly the expected total inventory cost. On the other hand, the expected logistics cost goes up as a result of the additional logistics movements. Overall, however, the expected total service lifecycle cost (i.e. aggregated inventory and logistics cost) is still about $11 \%$ lower compared to the scenario without any re-balancing as the increase in logistics cost is minor compared to the decrease in inventory cost.

Table 3: Optimised service contract with inventory re-balancing (p.a. = per annum)

\begin{tabular}{|c|c|c|c|c|c|c|c|c|c|c|c|}
\hline \multirow{4}{*}{ Optimised Stock Levels } & Airport & Part-10 & Part-09 & Part-08 & Part-07 & Part-06 & Part-05 & Part-04 & Part-03 & Part-02 & Part-01 \\
\hline & LAX & 5 & 3 & 4 & 2 & 1 & 2 & 1 & 1 & 1 & 0 \\
\hline & DFW & 3 & 2 & 3 & 1 & 0 & 1 & 0 & 1 & 0 & 0 \\
\hline & IAD & 4 & 3 & 3 & 2 & 0 & 1 & 0 & 1 & 1 & 0 \\
\hline \multicolumn{2}{|l|}{ Total Pool Stock } & 12 & 8 & 10 & 5 & 1 & 4 & 1 & 3 & 2 & 0 \\
\hline \multirow{2}{*}{ Rebalancing Policy } & Rebalance & Yes & Yes & Yes & Yes & No & Yes & No & No & No & No \\
\hline & Threshold & 1 & 1 & 1 & 1 & - & 1 & - & - & - & - \\
\hline \multicolumn{2}{|l|}{ Expected No. of Faults p.a. } & 38.66 & 29.46 & 19.35 & 9.56 & 3.85 & 3.38 & 2.58 & 1.86 & 0.96 & 0.30 \\
\hline \multirow{2}{*}{$\begin{array}{l}\text { Expected No. of No-fill } \\
\text { Events p.a. }\end{array}$} & Immediate Maintenance Demands & 0.06 & 0.49 & 0.00 & 0.14 & 2.40 & 0.05 & 1.57 & 0.11 & 0.27 & 0.30 \\
\hline & Total & \multicolumn{10}{|c|}{5.41} \\
\hline \multirow{2}{*}{ Service Level Achieved } & Immediate Maintenance Demands & $99.84 \%$ & $98.33 \%$ & $100.00 \%$ & $98.51 \%$ & $37.49 \%$ & $98.64 \%$ & $39.04 \%$ & $93.90 \%$ & $71.59 \%$ & $0.00 \%$ \\
\hline & Overall & \multicolumn{10}{|c|}{$95.08 \%$} \\
\hline \multirow{2}{*}{ Component Details } & MTBUR / QPA & 2500 & 3200 & 5000 & 10000 & 25000 & 27500 & 37500 & 50000 & 100000 & 300000 \\
\hline & Unit Price & $\$ 15,000$ & $\$ 150,000$ & $\$ 5,000$ & $\$ 45,000$ & $\$ 200,000$ & $\$ 8,000$ & $\$ 150,000$ & $\$ 60,000$ & $\$ 50,000$ & $\$ 45,000$ \\
\hline \multicolumn{2}{|c|}{ Expected Total Inventory Cost p.a. } & $\$ 36,000$ & $\$ 240,000$ & $\$ 10,000$ & $\$ 45,000$ & $\$ 40,000$ & $\$ 6,400$ & $\$ 30,000$ & $\$ 36,000$ & $\$ 20,000$ & $\$ 0$ \\
\hline \multicolumn{2}{|c|}{ Expected Total Inventory Cost across all Parts p.a. } & \multicolumn{10}{|c|}{$\$ 463,400$} \\
\hline \multirow{2}{*}{$\begin{array}{l}\text { Expected No. of Logistics } \\
\text { Movements p.a. }\end{array}$} & Deliveries & 0.06 & 0.44 & 0.00 & 0.14 & 1.50 & 0.04 & 1.10 & 0.11 & 0.27 & 0.00 \\
\hline & Rebalancings & 1.83 & 5.55 & 0.87 & 3.16 & 0.00 & 1.41 & 0.00 & 0.00 & 0.00 & 0.00 \\
\hline \multirow{3}{*}{ Expected Logistics Cost p.a. } & Deliveries & $\$ 63$ & $\$ 444$ & $\$ 0$ & $\$ 142$ & $\$ 1,496$ & $\$ 43$ & $\$ 1,096$ & $\$ 110$ & $\$ 270$ & $\$ 0$ \\
\hline & Rebalancings & $\$ 1,830$ & $\$ 5,548$ & $\$ 868$ & $\$ 3,164$ & $\$ 0$ & $\$ 1,412$ & $\$ 0$ & $\$ 0$ & $\$ 0$ & $\$ 0$ \\
\hline & Total & \multicolumn{10}{|c|}{$\$ 16,486$} \\
\hline \multicolumn{2}{|c|}{ Expected Total Service Lifecycle Cost p.a. } & \multicolumn{10}{|c|}{$\$ 479,886$} \\
\hline
\end{tabular}




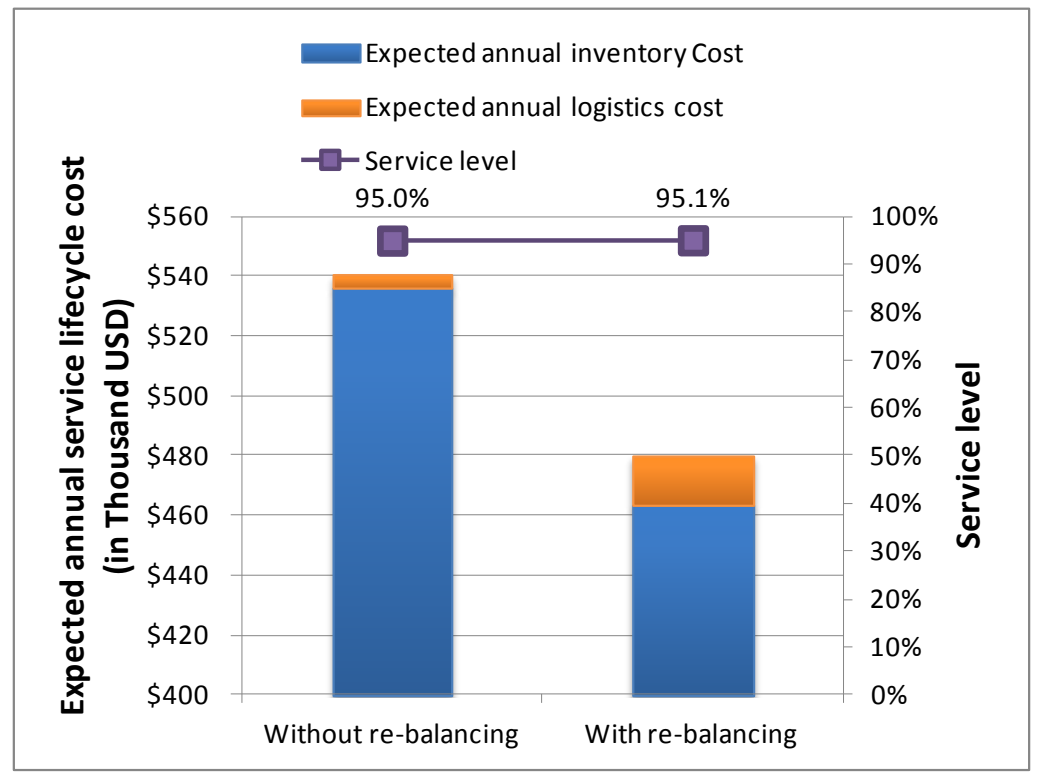

Figure 6: Expected annual service lifecycle cost with and without re-balancing as decision variable

\section{CONCLUSION}

In this paper, we demonstrated how simulation-based optimisation techniques can help to reduce the inventory cost for the multi-location inventory problem through a realistic case study. Specifically, we showed that the potential of pooling of inventory between inventory locations, depending on delivery time requirements, can be quantified, and that advanced logistics policies such as re-balancing between stockholding nodes can be used right away from the IP stage onwards to help reduce the total service lifecycle cost without increasing risk.

\section{REFERENCES}

Fung., S. H., C. F. Cheung, W. B. Lee, and S. K. Kwok. 2005. "A Virtual Warehouse System for Production Logistics." Production Planning and Control: The Management of Operations 16(6):597607.

Gunn., W.A. 1964. "Airline system simulation.” Operations Research 12(2):206-229.

Kilpi, J., and A.P.J. Vepsalainen. 2004. "Pooling of Spare Components Between Airlines." Journal of Air Transport Management 10:137-146

Landers., T.L., M.H. Cole, B. Walker, and R.W. Kirk. 2000. “The Virtual Warehousing Concept.” Transportation Research Part E 36(2):115-125.

Lee., L. H., H. C. Huang, C. U. Lee, E. P. Chew, W. Jaruphongsa, Y. Y. Yong, Z. Liang, C. H. Leong, Y. P. Tan, K. Namburi, E. L. Johnson, and J. Banks. 2003. "Discrete Event Simulation Model for Airline Operations: SIMAIR." In Proceedings of the 2003 Winter Simulation Conference, Edited by S. Chick, P. J. Sánchez, D. Ferrin and D. J. Morrice, 1656-1662. Piscataway, New Jersey: Institute of Electrical and Electronics Engineers, Inc.

Lendermann, P., B. P. Gan, N. Julka, A. Schirrmann, and H. Fromm. 2010. "Unlocking Value from Component Exchange Contracts in Aviation Using Simulation-based Optimisation." In Proceedings of the 2010 Winter Simulation Conference, Edited by B. Johansson, S. Jain, J. Montoya-Torres, J. Hugan, and E. Yücesan, 2034-2045. Piscataway, New Jersey: Institute of Electrical and Electronics Engineers, Inc. 
Lye, K.-W., and L.-P. Chan. 2007. "A Virtual Warehouse Simulation Tool for Aerospace Rotables Management." In Proceedings of the 2007 IEEE Aerospace Conference, 1-7. March 3-10, Big Sky, MT, USA.

MacDonnell, M., and B. Clegg. 2007. "Designing a Support System for Aerospace Maintenance Supply Chains." Journal of Manufacturing Technology Management 18(2):139-152.

MacDonnell, M., and B. Clegg. 2011. "A New Inventory Model for Aircraft Spare." In Service Parts Management: Demand Forecasting and Inventory Control, Edited by N. Altay and L. A. Litteral, 143-156. Springer Verlag Inc.

Rutledge, J. 1997. "Spare Parts: Cost Benefit Management.” Fast - Flight Airworthiness Support \& Technology 21:25-29.

\section{AUTHOR BIOGRAPHIES}

PETER LENDERMANN is the Co-Founder and CEO of D-SIMLAB Technologies, a Singapore-based company providing simulation-based decision support solutions and services to Aerospace, Semiconductor Manufacturing and other asset-intensive industries. Prior to this he worked at the Singapore Institute of Manufacturing Technology where he led the simulation-related research activities until spinning them off into D-SIMLAB Technologies. He has been engaged in the simulation community since the early 1990's. Peter holds a PhD in Applied High-Energy Physics from Humboldt- University in Berlin (Germany) and an MBA in International Economics and Management from SDA Bocconi in Milan (Italy). His email address is peter@d-simlab.com.

ANNAMALAI THIRUNAVUKKARASU is a Product Manager in D-SIMLAB Technologies (Singapore) where he is responsible for management of D-SIMLAB aerospace products. He graduated from Thiagarajar College of Engineering (India) with a BEng in Mechanical Engineering in 2006 and received his M.Sc. in Industrial and Systems Engineering from the National University of Singapore (NUS), Singapore, in 2009. His email address is annamalai@d-simlab.com.

MALCOLM YOKE HEAN LOW is currently the Vice President (Aerospace) of D-SIMLAB Technologies. Prior to this, he was an Assistant Professor in the School of Computer Engineering at the Nanyang Technological University (Singapore) where he led large-scale simulation and optimization research projects in the defence and maritime domains funded by the Singapore Ministry of Defence and the Maritime and Port Authority of Singapore. Malcolm holds a D.Phil. degree in Computer Science from Oxford University. His email address is malcolm@d-simlab.com.

LEON F. MCGINNIS is Professor Emeritus in the Steward School of Industrial and Systems Engineering at the Georgia Institute of Technology, where he continues to serve in leadership roles in the Manufacturing Research Center, the Model Based Systems Engineering Center, the Keck Virtual Factory Lab, and the Tennenbaum Institute for Enterprise Transformation. His area of interest is discrete event logistics systems, and his focus is on engineering methods for describing, analyzing, designing, and optimizing them. His email address is leon.mcginnis@isye.gatech.edu. 